Sharif University of Technology
Scientia Iranica
SCIENTIA $\quad \begin{gathered}\text { Transactions D: Computer Science \& Engineering and Electrical Engineering } \\ \text { whw.scientiairanica.com }\end{gathered}$

\title{
CMORC: Class-based Multipath On-demand Routing protocol for Cognitive radio networks
}

\author{
S. Rezaei* and A.M. Afshin Hemmatyar \\ Department of Computer Engineering, Sharif University of Technology, Tehran, Iran.
}

Received 26 July 2015; received in revised form 11 September 2016; accepted 7 January 2017

\section{KEYWORDS}

Cognitive radio;

Routing;

Multipath;

Optimization.

\begin{abstract}
Cognitive Radio (CR) technology enables Dynamic Spectrum Access (DSA) to improve the efficiency of under-utilized licensed bands and overcrowded unlicensed bands. However, providing an acceptable service for cognitive users requires more sophisticated approaches due to the existence of Primary Users (PU) with high priority over licensed bands. Additionally, it is of paramount importance that interference with PUs be reduced so that they can communicate without interruption. In order to meet requirements of users as much as possible and reduce interference with PUs, a new Class-based Multi-path Ondemand Routing protocol for Cognitive radio networks (CMORC) is proposed. CMORC is a multipath routing protocol, which defines two classes of routing to make delay or interference optimum. To the best of our knowledge, it is the first multipath protocol that takes into consideration both route discovery and rate allocation. CMORC finds the optimum sending rates for each path by solving an optimization problem for each routing class. Since solving these optimization problems is shown to be easy, CMORC is an efficient and practical solution for radio equipment with limited hardware. The simulation results reveal that CMORC outperforms the other recent multipath routing protocol, $\mathrm{D}^{2} \mathrm{CARP}$, in terms of interference, delay and packet delivery ratio depending on class type.
\end{abstract}

(C) 2017 Sharif University of Technology. All rights reserved.

\section{Introduction}

Increasing demands for bandwidth of wireless network due to recent technological advances and limited number of unlicensed bands have resulted in severe congestion over unlicensed bands. However, several underutilized licensed bands can be opportunistically used to satisfy user's demands, but they are not available because of the strict static allocation policy up until lately [1]. FCC has recently permitted unlicensed devices to use licensed bands, leading to a new technology

*. Corresponding author.

E-mail addresses: shrezaei@ce.sharif.edu (S. Rezaei);

hemmatyar@sharif.edu (A.M.Afshin Hemmatyar)

doi: $10.24200 /$ sci. 2017.4577 called Cognitive Radio (CR). CR technology improves the spectrum utilization by enabling the opportunistic use of temporarily idle licensed bands [2]. Although there has been an increasing trend of research on Cognitive Radio Networks (CRN) in the last decade, most of them have been mainly focused on physical and medium access issues.

Routing in traditional ad hoc networks has been studied even for complicated networks with multirate links [3]. However, routing is still in its nascent stage in multihop Cognitive Radio Ad Hoc Networks (CRAHN) [4]. Dynamic availability of licensed bands due to the presence of Primary Users (PU), licensed users, introduces new challenges to routing protocols. Many single-path routing protocols have been introduced to address these difficulties [5]. However, most of them ignore the importance of $\mathrm{PU}$ protection [2]. 
In CRAHN, PU protection as well as traditional performance metrics (e.g., delay, throughput, path length, etc.) are considered in routing protocols.

Previous studies have shown the efficiency of multi-path routing in wireless ad hoc networks [6]. In CRAHN, exploiting path diversity by employing multipath mechanism together with spectrum diversity may lead to an effective routing mechanism which can improve delay, reliability, throughput, interference, etc. However, only few studies have exploited path diversity in CRAHN. Besides, none of these studies addresses the issues of route discovery and rate allocation for multipath routing simultaneously. These two issues are somewhat connected and should be considered together to achieve the full benefit of multipath routing.

In this paper, we propose a class-based multipath routing protocol, which provides two classes of routing to minimize the end-to-end delay or probability of interference with PUs. To the best of our knowledge, this is the first paper that introduces a routing framework, which includes both route discovery and rate allocation. We show how customizing the route discovery with forwarding delay technique helps the rate allocation stage to perform efficiently. We also use a local channel selection mechanism to circumvent any unnecessary network overhead. Additionally, we propose a probabilistic method to estimate the overall end-to-end delay and interference which is used to formulate an optimization problem for each routing class. Then, we show that the number of variables in the optimization problems can be reduced without any negligible performance degradation so that it can be even solved by the exhaustive search method. Finally, we evaluate the performance of our proposed protocol through a set of simulations on NS-2.

The rest of this paper is organized as follows. In Section 2, we briefly describe the related research studies in this area. We specify network model and assumptions in terms of which our proposed protocol has been devised in Section 3. Section 4 describes the routing and channel selection stage. Section 5 gives a model to estimate routing parameters and explains how to assign a sending rate to each route based on the model. A comprehensive performance evaluation is carried out in Section 6. Finally, Section 7 concludes our work.

\section{Related work}

Multipath routing has been well studied in traditional wireless ad hoc networks, but there are only a few papers that explicitly explore the capabilities of multipath routing in CRAHN. In [7], Cognitive ad hoc Ondemand Distance Vector (CAODV) routing protocol with two different versions has been proposed to exploit path and spectrum diversity separately. The version exploiting path diversity is considered to be a multipath routing. The fixed channel assignment through the whole path in this version limits its application to some special cases and degrades its performance considerably. This also imposes more route failures due to the presence of PUs. $\mathrm{D}^{2} \mathrm{CARP}$ [8] improves CAODV by exploiting path and spectrum diversity simultaneously. However, $\mathrm{D}^{2} \mathrm{CARP}$ lacks the rate allocation stage and a specific end-to-end metric for its route discovery.

In [9], a multipath link-state routing protocol is proposed. Assuming that the network graph and frequency information of all nodes are always available, this protocol seeks to find the maximal set of the shortest cost paths using a modified version of Dijkstra's algorithm while minimizing interference. Nonetheless, that is not a reasonable assumption, especially in dynamic networks such as CRAHN. In [10], a multipath routing for underlay cognitive radio networks has been introduced. In this article, a specific distribution of primary and cognitive users is assumed which is not reasonable in CRAHN [8]. Furthermore, duplicating and coding techniques are used to enhance reliability, leading to overhead and throughput degradation.

In $[11,12]$, multipath routing protocols based on game theory have been proposed. These protocols assume that there are several paths already known by the source, and they model each path as a player and seek to find the best possible traffic assignment. However, these methods thoroughly neglect the route discovery stage and PU activity. In [4], a traffic assignment scheme is proposed. In this work, the authors have introduced a model based on queue theory for end-to-end delay of routes. They also derived an optimization problem from their delay model to minimize the total end-to-end delay and solved it by a gradient search method. In [4], similar to [11,12], it is also assumed that several paths are already available, and they do not address the route discovery process. Additionally, there is no comparison made with other multipath routing protocols in their simulations.

In [13], multi-channel multipath AODV has been proposed. This protocol is a modified version of AODV in which the route discovery messages are sent to all available channels. Despite the overhead of the protocol, the paper does not elaborate on how a route maintenance and traffic distribution among routes are being handled. Additionally, the proposed protocol is just compared with AODV, which is certainly not a suitable choice in CRAHN. In [14], a multipath routing protocol based on a centralized max-flow algorithm is proposed in which some strong and limiting assumptions have been made such as strong network connectivity, static topology during initialization process, and complicated time-slotted control channel.

In this paper, we proposed a class-based multi- 
path routing protocol to address both route discovery and rate allocation at the same time. The route discovery stage has been explicitly customized to simplify the rate allocation stage. Rate allocation stage has been developed to optimize sending rates on each path in order to minimize the overall end-to-end delay or interference. We also compared exhaustive method with genetic algorithm and interior point method to solve the optimization problem. Performance benefits of our routing protocol are shown by the comparison made with $\mathrm{D}^{2} \mathrm{CARP}$ through the simulations.

\section{Network model}

In our protocol, we assume that the network is composed of several cognitive users that are stationary or slowly moving. The primary users are also assumed stationary or slowly moving so that the history of channels stored locally by cognitive users could be used reliably. The location and frequency band of the primary users are unknown to the cognitive users, and the history of channels in each CU (Cognitive User) is simply obtained by locally sensing the channel. Note that in literature, both CU (Cognitive User) and SU (Secondary User) are used interchangeably, since only SUs have cognitive radio. We also assume that a Common Control Channel (CCC), free of any $\mathrm{PU}$ activities, is available in order to transfer control packets. Each CU has single or multiple cognitive radios to transfer data on licensed channels and an extra radio (without the cognitive ability) fixed to CCC. The PU activity is assumed to be a two-stage ON-OFF process, as shown in Figure 1 [15].

We assume that each node maintains the history of PU activities in order to compute the probability of channel availability. This probability can be statistically known or obtained by averaging the past channel history [2]. Denoting $\frac{1}{\alpha^{j, c h}}$ as an average idle time of PUs and $\frac{1}{\beta^{j, c h}}$ as an average active time of PU for $c h$ th channel computed by node $j$, the probability of channel availability of the $c h$ th would be [2]:

$$
P_{\mathrm{PU}}^{j, c h}=\frac{\beta^{j, c h}}{\alpha^{j, c h}+\beta^{j, c h}} .
$$

There are $n$ licensed bands shared among CUs and PUs, each of which has a different bandwidth denoted by $C_{c h}$ for the $c h$ th channel. Furthermore, we assume

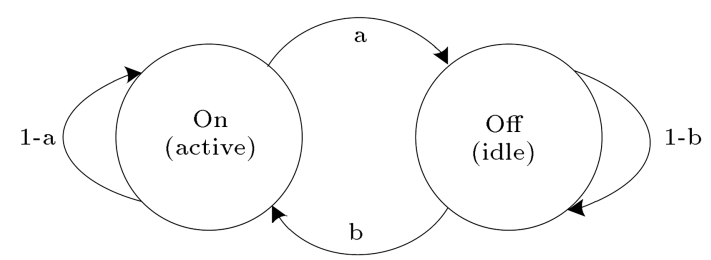

Figure 1. On-off model of PUs [15].

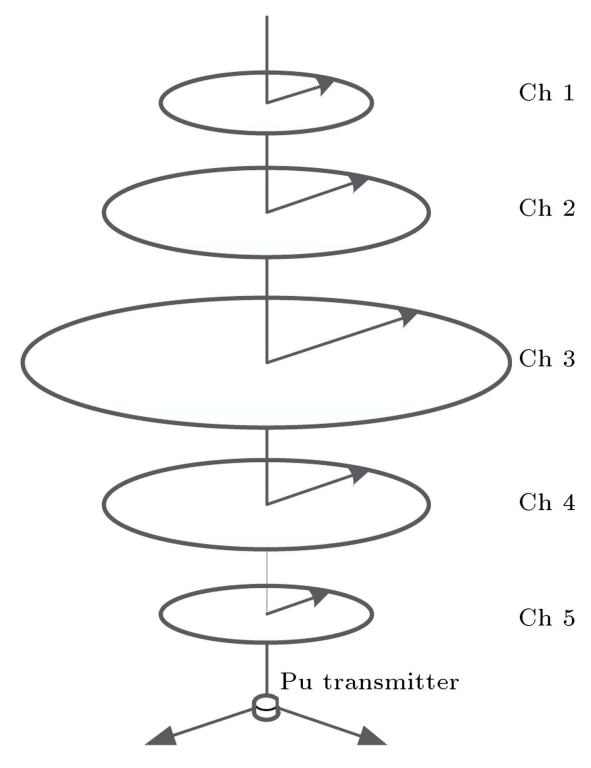

Figure 2. Adjacent channel interference effect [16].

that transmission in one channel causes an interference with adjacent channels. This interference effect is decreased proportional to frequency distance of these channels, and it is not effectual for channels that are more than 2 channel spacing away from the transmitter channel [16], as shown in Figure 2.

In our routing protocol, we consider two routing classes available for cognitive users. Generally speaking, the idea of defining different routing classes to satisfy user's various requirements is not new in cognitive radio networks. As an example, CRP routing protocol [2] has already defined two classes of routing as we have, yet the method to solve and satisfy these requirements is utterly different. These two classes of routing are introduced to satisfy different demands. Class I route seeks to optimally minimize the end-to-end delay of a session by finding paths and assigning an optimal sending rate to each available path. Class II route, however, seeks to minimize the probability of interference with PUs while neglecting delay and congestion entirely. Moreover, these routing classes assign an appropriate sending rate to each path so that the requested bandwidth of a source is satisfied. Needless to say that to achieve the best suitable solution within each routing class, not only the route discovery process, but also the rate allocation algorithm should be customized. Note that route and path are interchangeably used in this paper.

\section{Routing and channel selection stage}

In this section, we describe how a node selects a channel for each routing class and how the route discovery process manages to find several possible routes from the best to worst. At the end of this section, the route 
maintenance, which is performed due to route failure, is described as well.

\subsection{Channel selection and layer 2 considerations}

In CMORC protocol, each node selects its transmission channel by using channel information which is obtained locally. The only source-based parameter that affects channel decision of nodes is the routing class. Performing the channel selection locally in each node obviates the need for intermediate nodes to negotiate with the source. Hence, whenever the transmission channel of an intermediate node becomes unavailable due to PU activity, the intermediate node can change its channel immediately, such that the source does not even notice. As a result, the number of control packet transmissions and the time to select a channel for intermediate nodes are decreased.

The channel selection policy of each routing class should be different, since they seek to satisfy different requirements. Class I route minimizes the end-to-end delay which is inversely proportional to the available bandwidth. Each channel can be in one of these two states at each time: active or idle. With probability $1-P_{\mathrm{PU}}^{j, c h}$, the channel is in active state, which makes the channel unusable for CUs. In fact, CUs can use the channel only when it is on idle state with probability $P_{\mathrm{PU}}^{j, c h}$. Hence, denoting $L_{c h}$ as a load on the $c h$ th channel, the available bandwidth of each channel for transmission of node $j$ is $P_{\mathrm{PU}}^{j, c h} \cdot\left(C_{c h}-L_{c h}\right)$. Therefore, intermediate node $j$ selects the channel with maximum available bandwidth for class I route (important symbols and variables are summarized in Table 1). That is:

$$
\begin{aligned}
& \text { Data Channel } \text { Class }_{\mathrm{I}}= \\
& \max \left\{\sum_{i \in \forall \text { available_channels }} P_{\mathrm{PU}}^{j, i} \cdot\left(C_{i}-L_{i}\right)\right\} .
\end{aligned}
$$

Class II route, however, reduces interference with PUs as much as possible while delay and throughput is degraded. In order to meet that goal, the channel selection process always chooses a channel with the least PU activity among available channels regardless of its load, that is:

Data Channel Class $_{\mathrm{II}}=$

$$
\max \left\{\sum_{i \in \forall \text { available_channels }} P_{\mathrm{PU}}^{j, i}\right\} .
$$

Layer 2 module of the CMORC routing protocol is assumed to provide channel set and history of all available channels, in addition to channel selection. Channel set is defined as a sorted list of all channels

Table 1. Description of symbols.

\begin{tabular}{ll}
\hline \multicolumn{1}{c}{ Symbol } & \multicolumn{1}{c}{ Description } \\
\hline$r_{i}$ & Sending rate of the $i$ th path \\
$D_{P}$ & Propagation delay \\
$c h \_s e t_{j}^{\mathrm{I} / \mathrm{II}}$ & Sorted set of channels at $j$ th node from best to worst \\
$\varphi^{\mathrm{I} / \mathrm{II}}(c h)$ & Set of channels that are better than $c h$ th channel \\
$|x|$ & Number of elements in the set $X$ \\
$P_{\mathrm{PU}}^{j, c h}$ & Probability of availability of $c h$ th channel at $j$ th node \\
$D_{q}^{j, c h}\left(r_{i}\right)$ & Queuing delay of $c h$ th channel at $j$ th node for $r_{i}$ rate \\
$B W_{\mathrm{req}}^{(S, D)}$ & Requested bandwidth of the source to destination \\
$\frac{1}{\alpha^{j, c h}}$ & Average PU idle time in $c h$ th channel at $j$ th node \\
$\frac{1}{\beta^{j, c h}}$ & Average PU active time in chth channel at $j$ th node \\
$L_{c h}^{j}$ & Traffic load of $c h$ th channel at $j$ th node \\
$D_{S e}$ & Sensing delay of CUs \\
$D_{s w}$ & Channel switching delay \\
$\omega_{j}(c h)$ & Probability of switching to chth channel at $j$ th node \\
$\delta_{\mathrm{I} / \mathrm{II}}$ & Forwarding delay constant for the I/IIth class \\
$C_{c h}$ & Bandwidth of chth channel \\
$\rho_{j}^{\mathrm{I} / \mathrm{II}}(c h)$ & Probability of selecting the chth channel at $j$ th node \\
paths $(S, D)$ & Set of all paths discovered from $S$ to $D$ \\
links $(i)$ & Set of all links in $i$ th path \\
int ${ }_{i}$ & Total interference of $i$ th path \\
\hline
\end{tabular}


from the best to worst considering the routing class, which can be easily obtained by using channel selection equations (Eqs. (2) and (3)). The channel history consists of the probability of channel availability and the load on that channel. The routing and the channel selection modules of layer 2 use these pieces of information.

The method with which a node collects PU activities and channel load is of a great importance. In the case where a node has multiple radios, layer 2 module of each radio can simply sense and collect these data. However, in the case where a node has a single cognitive radio, it is not feasible for a node to sense all the available channels simultaneously. One solution is to let adjacent nodes share their information periodically in CCC. In any case, details and problems of layer 2 are out of the scope of this paper.

\subsection{Route discovery}

CMORC is an on-demand routing protocol based on DSR. When a source needs to communicate with the destination and no routing information is available, it floods route request (RREQ) packet to the whole network over the CCC. Intermediate nodes forward these RREQ packets upon receiving them for the first time. As a result, the destination gets several RREQs, indicating different routes. However, this basic route discovery process of DSR is not efficient to find as many node-disjoint routes as possible. Previous studies on multipath routing in ad hoc networks have shown that this basic procedure only discovers routes that are mostly overlapped due to the elimination of duplicate RREQ [17]. Hence, CMORC allows intermediate nodes to forward duplicate RREQs only if these two criteria are met:

1. The path length of the route in the RREQ should not be longer than the previous RREQs;

2. The first hop of the route (the next hop of the source) should not be the same as the previous RREQs.

The first condition prevents not only flooding of longer routes, but also interminable flooding of RREQ packets. The second condition prevents overlapped routes flooding to the entire network [17]. That is, most of the routes that reached the destination are node-disjoint.

When the destination receives the first RREQ, it generates Route Reply (RREP) packet, containing route information, and sends it back to the source. Upon receiving next RREQs, the destination checks whether the route is node-disjoint compared to all routes already sent to the source. If the new route is node-disjoint, the destination sends RREP back to the source and informs the new route. Otherwise, that RREQ is discarded.
This strategy of selecting node-disjoint routes is crucially dependent on the order of routes in which they are received. As an example, if a short path with the worst possible channels is received first, all better routes with at least one mutual intermediate node with the first route are discarded by the destination. Therefore, changing the order of RREQ arrival to obtain the better routes earlier increases the overall quality of routes in the source. Namely, the sooner the route is received, the less probable it is discarded.

CMOCR intentionally imposes forwarding delay proportional to the quality of the node and its channels in order that routes with better nodes and channel condition can be received earlier. This method, sometimes called jitter mechanism, has been studied in ad hoc networks recently [18]. Perhaps, this forwarding delay should be different for two routing classes since they satisfy different goals. For class I route, which aims to reduce end-to-end delay, forwarding delay is proportional to the load of the selected channel. That is, the routes that pass through channels with heavier traffic load end up having higher end-to-end delay. Hence, it is reasonable to impose greater forwarding delay for nodes of heavier channel load. Since the selected channel of an intermediate node is not fixed and may change during the transmission, the forwarding quality of that node depends on probability of selecting each channel, which is computed probabilistically. To compute the forwarding delay of class I route, CMORC multiplies channel selection probability of each channel by its load:

$$
\begin{gathered}
\operatorname{RREQ}_{\text {delay_Class }_{\mathrm{I}}}^{j}=\sum_{c h=1}^{\left|c h \_\operatorname{set}_{j}^{\mathrm{T}}\right|} \delta_{\mathrm{I}} \cdot L_{c h}^{j} \\
\cdot\left\{\prod_{k \in \varphi^{\mathrm{I}}(c h)}\left(1-P_{\mathrm{PU}}^{j, k}\right)\right\} \cdot P_{\mathrm{PU}}^{j, c h} .
\end{gathered}
$$

As we mentioned before, $c h \_$set $_{j}^{\mathrm{I}}$ is a sorted list of all channels from the best to worst for class I route that contains $\left|c h \_\operatorname{set}_{j}^{\mathrm{I}}\right|$ elements, and $\varphi_{j}^{\mathrm{I}}(c h)$ returns back a set of channels that is better than the chth channel from $c h \_s e t_{j}^{\mathrm{I}}$. So, the product on the formula computes the probability that all channels better than the chth one are used by PUs, that is, the product computes the probability of selecting the chth channel by layer $2 . \quad \delta_{\mathrm{I}}$ is a constant, indicating the amount of forwarding delay expressed in units of second that depends on the network's scale and route discovery timeout. Therefore, the forwarding delay is much longer for the nodes with heavier traffic load on their channels. Choosing a big value for $\delta_{\mathrm{I}}$ increases the time of route discovery process, and choosing a small value for $\delta_{\mathrm{I}}$ prevents the forwarding delay mechanism from operating efficiently. 
CMORC computes forwarding delay for class II routes as follows:

$$
\begin{gathered}
\operatorname{RREQ}_{\text {delay_Class }_{\mathrm{II}}}^{j}=\sum_{c h=1}^{\mid \text {ch_set }_{j}^{\mathrm{II} \mid}} \delta_{\mathrm{II}} \\
\cdot\left\{\prod_{k \in \varphi^{\mathrm{I}}(c h)}\left(1-P_{\mathrm{PU}}^{j, k}\right)\right\} \cdot P_{\mathrm{PU}}^{j, c h} .
\end{gathered}
$$

$\delta_{\mathrm{II}}$ is a forwarding delay constant of class II route. Since the aim of class II route is to minimize interference, the load on the channel is entirely omitted. Therefore, the probability of channel selection, which depends on PU activity, is the only variable that affects the forwarding delay of class II route. That is, nodes with channels that are idler (from PU) forward their RREQ packet with less delay. Hence, the routes passing through idler channels are received earlier by the destination.

As mentioned earlier, CMORC has a rate allocation stage performed in the source that needs extra data to be collected in the route discovery stage. In addition to the node IDs added to the RREQ packets by intermediate nodes to indicate the route, which is a part of DSR routing protocol, CMORC adds extra information to RREQs, used later in the source to perform rate allocation stage. The MAC layer mainly provides this information. It consists of:

1. Propagation delay of the last link;

2. Probability of the availability of all channels;

3. Load on all channels.

Although, theoretically speaking, there is no limitation on the amount of data carried by RREQ packets, it is not possible to put all channel statistics of all intermediate nodes into a single RREQ packet. Hence, intermediate nodes just put statistics of the top 3 channels in RREQs. This number of channel statistics added to RREQ packets can vary depending on the scale of the network. In this paper, however, we only use statistics of 3 channels.

Having received the first RREQ packet, the destination sends back a RREP packet to the source, containing all the intermediate node information, and registers the new route in its routing table. When the destination receives the next RREQ packets, it checks whether the new route has any mutual intermediate node with the previous routes. If so, the RREQ is discarded and route is avoided. But, if the new route is completely node-disjoint compared to previously received routes, the route is registered in the destination routing table and a corresponding RREP is sent back to the source. Checking the existence of mutual intermediate nodes, destination guarantees a set of node-disjoint paths at source.

\subsection{Route maintenance}

In cognitive radio networks, two sources of route failure are node movements and PU appearance. Routing protocols that rely on channels, to which source or the routing discovery process is assigned, usually fail in recovering from any PU appearance. At best, the intermediate node sends a route error (RERR) packet to the source and waits for the source to reply, or the route becomes useless. In contrast, $\mathrm{PU}$ appearance is dealt with locally in CMORC by choosing the best available channel from the channel set. Hence, the route is recovered safely without any delay or network overhead. When the route fails due to node movements, nodes that noticed the disconnectivity in the path send a RERR to the source to inform. Afterward, the source removes that route from its routing table and performs the rate allocation of the remaining routes. If the requested bandwidth of the source is not satisfied with the remaining routes, or the number of routes is less than two, the route discovery process will be called to find more routes.

\section{Rate allocation stage}

When the route discovery stage is finished, the source has several paths to the destination. In multipath routing protocols, like CMORC, distribution of traffic among available paths has a significant effect on the performance. Hence, rate allocation stage is an essential part of a multipath routing protocol.

In this section, we introduce an optimization problem for each routing class to get the optimum sending rate of available paths. Practical methods of solving these optimization problems are also described that impose less computational overload than prevalent methods.

\subsection{Problem formulation}

\subsubsection{Class I routes}

In class I routes, where the aim is to minimize the endto-end delay, CMORC distributes traffic in a way that the congestion and packet loss are avoided in the paths. CMORC also sends more traffic through the path with more available capacity to reduce the overall end-toend delay. In addition, since the available capacity of a path is related to the PU activities, modeling the end-to-end delay, in the way that it implicitly contains channel history, will reduce probability of interference with PUs as well.

At the end of the route discovery stage, the source has several node-disjoint paths to the destination. Since the routes are node-disjoint, the end-to-end delay of each path is almost independent of the other paths. We assumed that the two routes, which are sending at the same channel, are not close enough to interfere. Therefore, the queuing delay of the $j$ th node over the 
chth channel can be obtained by knowing the channel load and sending rate of that path:

$$
D_{q}^{j, c h}\left(r_{i}\right)=\frac{1}{\left(C_{c h}-L_{c h}^{j}\right)-r_{i}}
$$

where $r_{i}$ denotes the sending rate on the $i$ th path, and $D_{q}^{j, c h}\left(r_{i}\right)$ represents the queuing delay of $\mathrm{M} / \mathrm{M} / 1$ queue, assuming that arrival process is Poisson [19]. Note that M/M/1 queuing model does not accurately model the wireless network. However, since CMORC just needs a basic estimation to compare the routes, this model is used to keep the computation as simple as possible. Because the channel over which the intermediate node forwards traffic may be changed during the session, the source cannot use a specific channel to obtain the queuing delay. Hence, the source gets the probabilistic queuing delay of the node by multiplying the probability of selecting that channel by its queuing delay:

$$
\sum_{c h=1}^{\left|c h \_\operatorname{set}_{j}^{\mathrm{T}}\right|} \rho_{j}^{\mathrm{I}}(c h) \cdot D_{q}^{j, c h}\left(r_{i}\right) .
$$

Henceforward, we use $\rho_{j}^{\mathrm{I} / \mathrm{II}}(c h)$ to denote the probability of selecting the chth channel by the $j$ th node for class I/II route:

$$
\rho_{j}^{\mathrm{I} / \mathrm{II}}(c h)=\left\{\prod_{k \in \varphi^{\mathrm{I} / \mathrm{II}}(c h)}\left(1-P_{\mathrm{PU}}^{j, k}\right)\right\} \cdot P_{\mathrm{PU}}^{j, c h} .
$$

In addition to the queuing delay, there are some other factors that impose delay such as propagation time, sensing delay, and switching delay. Sensing delay refers to the time period when cognitive users become silent so as to monitor PU activities. Sensing time entirely depends on layer 2 protocol, and it may be changed or omitted from one protocol to another. Switching delay refers to the time needed for a cognitive user to change its frequency band or channel. In networks where cognitive users have multiple radios (when the number of available channels and radios are equal), there is no switching delay (e.g., $\mathrm{D}^{2} \mathrm{CARP}$ [8]). Consequently, the total link delay can be obtained as follows:

$$
D_{S e}+\sum_{c h=1}^{\left|c h \_\operatorname{set}_{j}^{\mathrm{T}}\right|} \rho_{j}^{\mathrm{I}}(c h) \cdot\left\{D_{q}^{j, c h}\left(r_{i}\right)+D_{P}+\omega_{j}(c h) \cdot D_{s w}\right\}
$$

where $D_{S e}, D_{P}$, and $D_{s w}$ denote sensing delay, propagation delay, and switching delay, respectively. Sensing delay is the only parameter assumed independent of the selected channel. Switching delay, however, depends on the selected channel. Since the source does not know the selected channel of intermediate nodes, it computes the switching delay probabilistically. Hence, the source multiplies the probability of switching the channel by ch, denoted by $\omega_{j}(c h)$, to the switching delay. Suppose that two consecutive nodes, represented by $j-1$ and $j$, are on the same path. If transmission channel of $j-1$ (receiving channel of $j$ ) is the same as that of $j$, node $j$ does not need to change its channel. Therefore, the probability of channel switching is equal to the probability that node $j-1$ chooses any channel rather than $c h$ :

$$
\omega_{j}(c h)=1-\left\{\prod_{k \in \varphi^{\mathrm{I}}(c h)}\left(1-P_{\mathrm{PU}}^{j-1, k}\right)\right\} \cdot P_{\mathrm{PU}}^{j-1, c h} .
$$

So far, we described a formula to get probabilistic link delay that includes $\mathrm{M} / \mathrm{M} / 1$ queuing delay. Considering that the departure process of $M / M / 1$ queue has a Poisson distribution [19] and the arrival rate is less than the departure rate, we can simply assume that the arrival rate of all intermediate nodes in the path is $r$. Although it is not completely true as long as queueing theory is concerned, it is the simplest estimation that makes the comparison of the routes possible. Note that CMORC does not model the delay accurately. It uses a simple and computationally-feasible formula to compare routes so as to distribute traffic better than the blind, equal-rate, round-robin method. Hence, endto-end delay of a route is the sum of delay of all links throughout the path. Consequently, the overall end-toend delay of the session which, includes several paths, can be estimated by multiplying the sending rate of each path by its delay. Therefore, the optimization problem for class I route is as follows:

$$
\begin{gathered}
\min \sum_{i \in \operatorname{paths}(S, D)} r_{i} \cdot \sum_{j \in \operatorname{links}(i)}\left\{D_{S e}+\sum_{c h=1}^{\left|c h_{-} \operatorname{set}_{j}^{\mathrm{T}}\right|} \rho_{j}^{\mathrm{I}}(c h)\right. \\
\left.\cdot\left\{D_{q}^{j, c h}\left(r_{i}\right)+D_{P}+\omega_{j}(c h) \cdot D_{s w}\right\}\right\}
\end{gathered}
$$

subject to:

$$
\begin{aligned}
& \sum_{i \in \operatorname{paths}(S, D)} r_{i}=B W_{\text {req }}^{(S, D)}, \\
& r_{i}<\min _{\forall j \in \operatorname{links}(i)}\left\{C_{c h}-L_{c h}\right\}, \quad \forall i \in \operatorname{paths}(S, D) \\
& r_{i} \geq 0, \quad \forall i \in \operatorname{paths}(S, D)
\end{aligned}
$$

The first constraint indicates that the total sending rates of all paths must satisfy the source bandwidth requirement. The second constraint limits the maximum value of sending rate of the $i$ th path to the 
available capacity of its worst channel. Not only does this constraint makes the problem convex, which will be shown later, but also it is a necessary condition for the assumption that the arrival rates of all intermediate nodes of the $i$ th path are all equal to $r_{i}$. Finally, the third constraint indicates that the sending rates cannot be negative.

\subsubsection{Class II routes}

The aim of class II route is to minimize the total interference with PUs. However, calculating the accurate interference needs extra information such as location of PU receivers, which is not provided in all networks. Hence, the only statistical data available in cognitive users to estimate the total interference is the probability of channel availability $\left(P_{\mathrm{PU}}^{j, c h}\right)$ which is obtained by monitoring PU activities. Generally speaking, channels observed to have fewer PU activities are most likely to be free of any adjacent PU receivers. As a result, CMORC directly uses the probability of channel availability to estimate the total interference.

In class II route, layer 2 module always selects the channel with the highest probability of channel availability $\left(P_{\mathrm{PU}}^{j, c h}\right)$ to reduce the interference. Hence, the interference is proportional to $1-P_{\mathrm{PU}}^{j, c h}$ and since the transmission channel is not fixed during a session, probability of selecting the channels $\left(\sum \rho_{j}^{\mathrm{II}}(c h)\right)$ is used instead of a fixed channel. Consequently, the path interference, which is the sum of the interference of all links, is estimated as follows:

$$
\text { int }_{i}=\sum_{j \in \operatorname{links}(i)}\left\{1-\sum_{c h=1}^{\mid c h \_ \text {set }_{j}^{\mathrm{II} \mid}} \rho_{j}^{\mathrm{II}}(c h)\right\}
$$

As a result, the total interference can be estimated by multiplying the sending rate of each path by its path interference. Class II optimization problem is as follows:

$$
\min \sum_{i \in \operatorname{paths}(S, D)} r_{i} \cdot \text { int }_{i}
$$

where the constraints are similar to class I optimization constraints. Note that CMORC estimates the end-toend delay and interference just to compare the routes and assign sending rates. Therefore, the real value of these parameters in the network might be slightly different from the estimated values.

\subsection{Reduction of optimization variables}

Before we represent a practical method to solve these optimization problems, the number of optimization variables and complexity of these two problems are analyzed. The number of variables in an optimization problem is extremely important. In our optimization problem, the only variables are the sending rates.
Hence, the number of variables is equal to the number of routes that depends on the scale of the network and number of nodes.

Since CMORC uses the forwarding delay technique in route discovery stage, routes that are received earlier are expected to outperform the others. That is, sending rate of these routes is expected to be greater. In order to observe the validity of this assertion, over hundred simulations are conducted and solved by the interior point method [20] (the detail of simulations is discussed later in Section 6). The result of the simulation is shown in Figure 3.

As shown in Figure 3, the first route has the most portion of the assigned rate. In other words, the first path satisfies almost half of the requested bandwidth. The second and third paths on average satisfy $24 \%$ and $13 \%$ of the requested bandwidth. Therefore, it clearly shows that the routes discovered earlier are always better, and the efficiency of the forwarding delay technique is proven. Furthermore, this result shows that the fourth and fifth routes transmit less than $10 \%$ of the requested bandwidth, and the rate allocated to next routes is almost negligible. Hence, the source can always solve the optimization problem for only the first three or five routes without any significant performance degradation. This helps the source to solve the optimization problems faster. Besides, the source knows when to stop waiting for route discovery process and drop other useless routes. In other words, the source starts to find the solution to the optimization problem whenever it receives 3 routes. Consequently, the node deals with less memory and computation overhead.

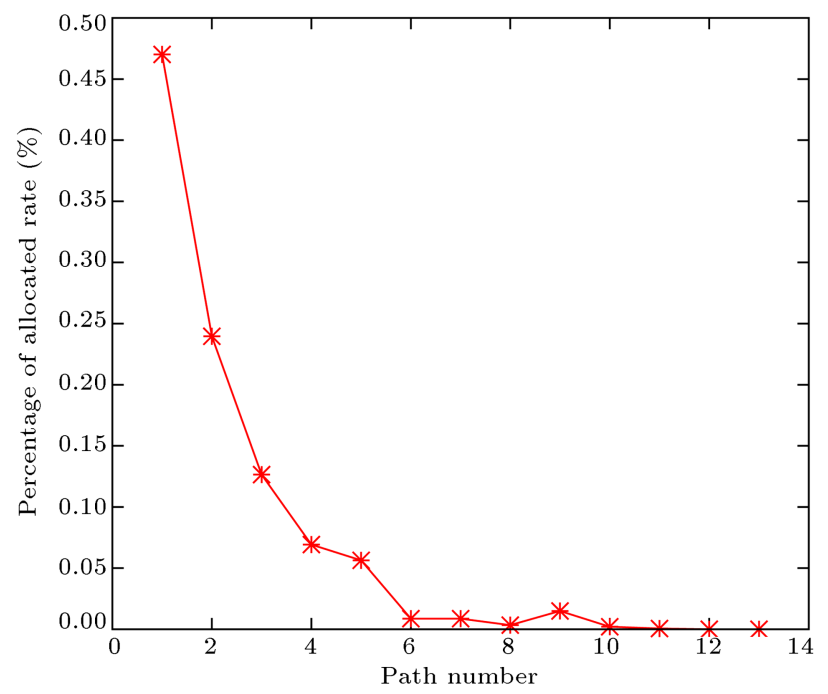

Figure 3. Percentage of allocated rate versus path number. Simulations show that by using forwarding delay technique and solving the optimization problem, almost all portions of the sending rates are allocated to the first five routes. Hence, one can ignore other routes to simplify the optimization problem. 


\subsection{Solving optimization problems}

\subsubsection{Class I optimization}

As we mentioned earlier in Section 5.2, the only variables in the objective function of class I optimization problem are the sending rates. Besides, the delay of each path is estimated by its sending rate regardless of the sending rates of other paths. Since the sum of multiple convex function is a convex function [20], proving the convexity of end-to-end delay of any path is sufficient to show the convexity of the objective function. Suppose that $f\left(r_{i}\right)$ represents the end-toend delay of the $i$ th path using Eq. (9). $f\left(r_{i}\right)$ is defined for all $0 \leq r_{i}<\min _{\forall j \in \operatorname{links}(i)}\left\{C_{c h}-L_{c h}\right\}$, which is indicated by the second and third constraints. Hence, $f\left(r_{i}\right)$ is continuous and differentiable on its domain. By taking the second derivative of $f\left(r_{i}\right)$, it is easy to see that its second derivative is positive in its domain. Therefore, $f\left(r_{i}\right)$ and the objective function are convex functions. In addition, all constraints of the optimization problem are clearly linear. Therefore, class I optimization problem is convex.

Not only can heuristic algorithms (e.g., genetic algorithm, simulated annealing) find an acceptable solution to our small-scaled problem, but also, due to the convexity of the problem, many mathematical methods (e.g., interior point, subgradient) can solve the problem as well. Mathematical methods usually need gradient or hessian of the function, which is not always easy to compute. Additionally, they impose complicated mathematical computation which is not a good choice for wireless nodes with limited memory and computation capacity. Heuristic algorithms also need a lot of iteration to find a near-optimal solution. Hence, a better algorithm for our small-scaled problem is needed to be devised to compute faster with less complexity.

Since we have already reduced the number of variables of the optimization problem to 3 or 5 , it seems reasonable to study the performance of exhaustive method with other possible methods. The variables

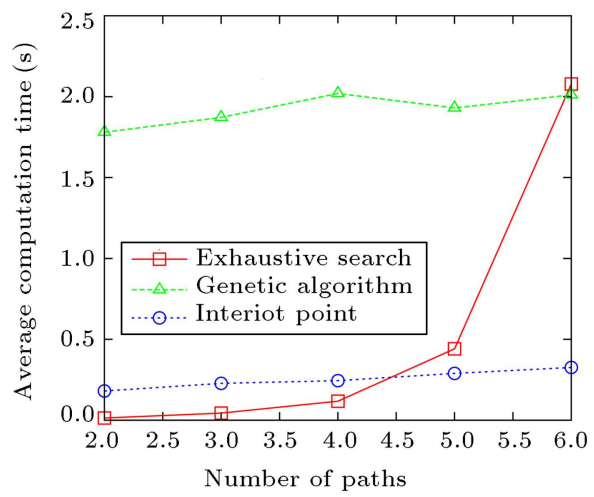

(a) of the optimization problem are real numbers in a particular range. In order to search the whole solution space, we have to make them discrete. As a result, we divide the whole possible range of each variable into 10 equal segments and use the first number in that range to represent the discrete scale. As an example, $r_{i}$ in range $[0,2) \mathrm{Mbps}$ is transformed to the discrete sequence of $\{0,0.2,0.4,0.6,0.8,1,1.2,1.4,1.6,1.8$, $2\}$.

In order to evaluate the performance of exhaustive search, we conducted a series of simulations to show the effect of number of variables (number of considered paths) on the computation time and quality of solution. In these simulations, interior point method and genetic algorithm are compared with exhaustive search method. The result of the simulations is shown in Figure 4. In Figure 4(a), it is shown that the average computation time of exhaustive search method, when fewer than 5 paths are considered, outperforms the other methods, while the optimized value of its solution is almost equal to the interior point method (Figure 4(b)). Genetic algorithm is always the worst method in both computation time and optimal solution. Hence, the exhaustive search, when the number of considered paths is lower than 5 paths, is a better choice since it does not need a lot of memory and extra computation (for obtaining gradient or hessian). However, one can also use mathematical methods, since the search space is very small and no meaningful difference exists. In fact, we are just providing a method that is as good as the others at worst, yet it does not need much memory and complicated mathematical computation of hessian or gradient, etc.

\subsubsection{Class II optimization}

The optimization problem of class II is much easier to solve. Objective function of this optimization problem (Eq. (16)) is based on product of interference of paths (int ${ }_{i}$ ) and their sending rate. The interference of

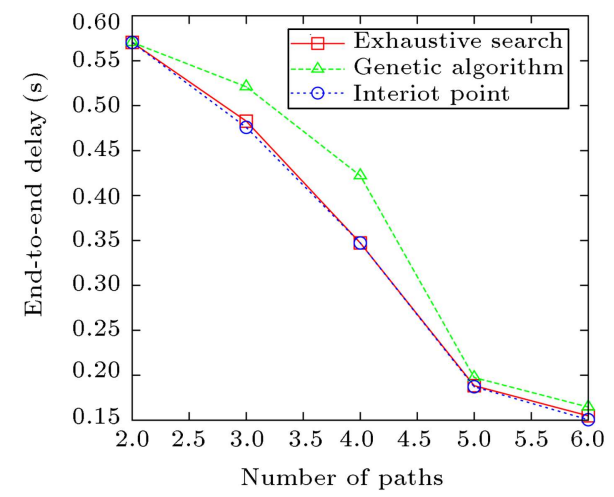

(b)

Figure 4. Comparison of optimization algorithms for class I routes. Number of paths versus (a) average computation time and (b) end-to-end delay. 


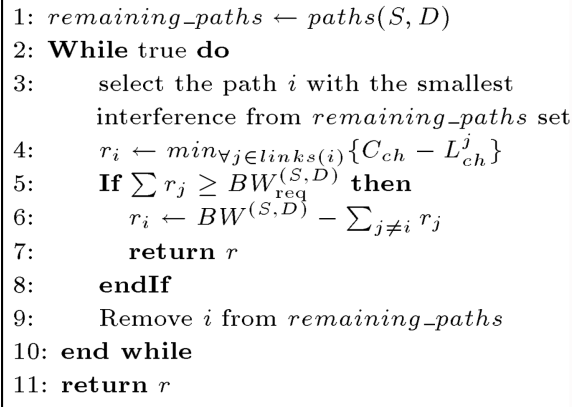

Algorithm 1. Greedy approach to solve the class II optimization problem.

a path, however, is independent of its sending rate. Hence, the objective function is clearly linear and int ${ }_{i}$ is a constant for $r_{i}$. Constraints of this optimization are the same as class I optimization constraints, so they are also linear. Since the whole problem is linear, it can be easily solved by linear optimization algorithms, such as Simplex. CMORC, however, uses a greedy algorithm shown in Algorithm 1 with the complexity of $O(n)$ where $n$ is the number of routes.

Greedy approach sorts all available paths by their interference and puts them in the remaining_paths set. Then, it chooses the path with the smallest interference from the remaining paths, assigns maximum possible rate to it, and removes it from the remaining_paths. Then, it chooses the next path from the remaining paths and goes on until the requested bandwidth is satisfied.

It is easy to show that the greedy approach of Algorithm 1 always finds the global optimum solution to class II optimization problem.

\section{Performance evaluation}

In this section, we study the performance of our proposed routing protocol with $\mathrm{D}^{2} \mathrm{CARP}$ [8] which is a reactive multipath routing protocol for cognitive radio networks. We implemented CMORC on Network

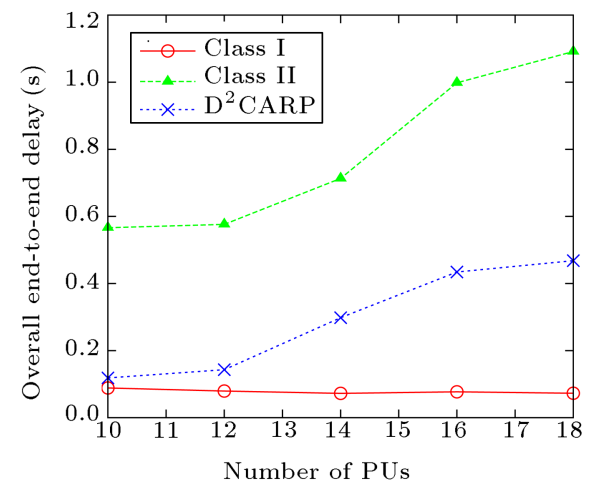

(a) simulator 2 (NS-2) with 2 routing classes. To carry out a fair evaluation, cognitive nodes in our simulations are implemented to have multiple radios since $\mathrm{D}^{2} \mathrm{CARP}$ is unable to work efficiently in single radio environment.

\subsection{Simulation parameters}

In our simulations, 100 cognitive users are randomly deployed in a square region whose size has been set to satisfy node density of 400 nodes $/ \mathrm{km}^{2}$. There are 11 different channels with the same bandwidth, for simplicity, and one of them is assumed to be a CCC and free of PU activity for CMORC. The transmission range of cognitive users is $120 \mathrm{~m}$. Forwarding delay constants of classes I and II routes $\left(\delta_{\mathrm{I} / \mathrm{II}}\right)$ are $0.001 \mathrm{~s}$ and $0.005 \mathrm{~s}$, respectively. In our simulation, CMORC uses only 5 paths to perform rate allocation stage for class I route, and since the optimization problem of class II is easier to solve, CMORC computes rate allocation of 10 paths for this class. Other simulation parameters are the same as $\mathrm{D}^{2} \mathrm{CARP}$ [8].

\subsection{End-to-end delay and packet delivery ratio} In this section, we study the effect of different parameters on the end-to-end delay and Packet Delivery Ratio (PDR). In Figure 5, we observed that increasing the number of PUs has no significant effect on the endto-end delay and PDR of class I routes, and this class always outperforms the others as far as delay and PDR are concerned. Class II, on the other hand, shows the worst end-to-end delay and PDR, since it transmits the traffic through probably longer paths and imposes congestion on those paths and channels. Both class II and $\mathrm{D}^{2} \mathrm{CARP}$, unlike class I, incur an increase in endto-end delay and decrease in PDR when the number of PUs is increased.

Figure 6 demonstrates the effect of increasing the number of flows on the end-to-end delay and PDR. The requested bandwidth of each flow is $0.54 \mathrm{Mbps}$. As it is expected, class I always outperforms the others, but the difference of these protocols and classes is negligible when there are just a few flows. That is

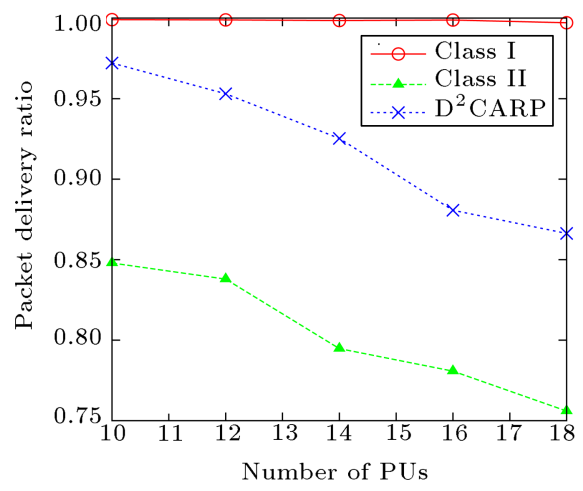

(b)

Figure 5. The number of PUs versus (a) overall end-to-end delay, and (b) packet delivery ratio. 


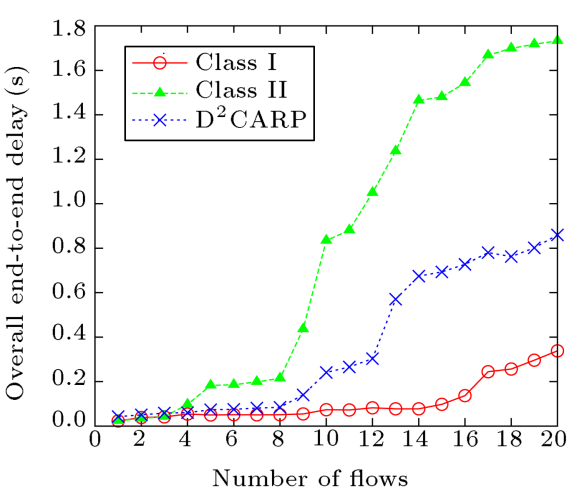

(a)

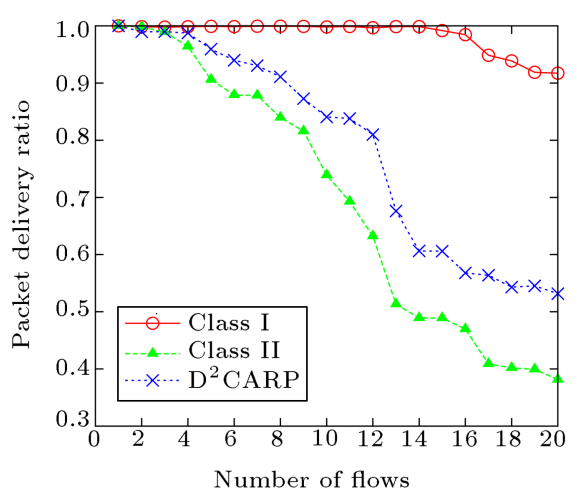

(b)

Figure 6. The number of flows versus (a) overall end-to-end delay, and (b) packet delivery ratio.

because the traffic is too low to make the channel congested. Unlike Figure 5, performance of class I slowly decreases although it still degrades slower than the others. Class II and $\mathrm{D}^{2} \mathrm{CARP}$, however, show almost the same behavior as in Figure 5 when the number of PUs is increased.

\subsection{Probability of interference}

In this section, we exclusively study the effect of routing protocols on total interference. To measure the probability of interference, we measure the probability of collision with cognitive users in the active period of PUs. The total interference might be different for various MAC and physical layer designs since channel history, sensing accuracy, and channel selection depend on these layers, but the comparison is reliable as long as these layers are the same for the compared protocols.

Figure 7(a) shows the effect of increasing the number of PUs on the total interference. It is observed that class II avoids interference as much as possible and the interference is almost zero when there are fewer than 16 PUs. Besides, it seems probably inevitable to interfere with $\mathrm{PUs}$ when the number of PUs passes a threshold. Interestingly, Figure 7 demonstrates that the probability of interference of class I routes is less than $\mathrm{D}^{2} \mathrm{CARP}$ routes most of the times. In other words, both routing classes of CMORC incur less interference than $\mathrm{D}^{2} \mathrm{CARP}$. The primary reason for this is that the rate allocation formulas of class I indirectly utilize channel history in its computation. Hence, since the channels with less probability of channel availability impose more delay, class I of CMORC implicitly selects channel and path with less interference. Figure $7(\mathrm{~b})$ also shows that the total interference of $\mathrm{D}^{2} \mathrm{CARP}$ increases faster than both routing classes of CMORC. In contrast, class II total interference increases extremely slowly that is almost negligible.

\subsection{Routing overhead}

Although both CMORC and $\mathrm{D}^{2} \mathrm{CARP}$ are on-demand routing protocols, they are based on different routing protocols. CMORC is based on DSR, but $\mathrm{D}^{2} \mathrm{CARP}$ is based on AODV. Hence, they apply somewhat different methods to pass information and indicate a route. CMORC puts all node IDs of the route in the routing header of the packet so that intermediate nodes know the path and the next hops. On the other hand, intermediate nodes in $\mathrm{D}^{2} \mathrm{CARP}$ store an entry in route discovery stage that shows the next hop of each flow, so they do not need any extra information in the header of packets. Therefore, the routing

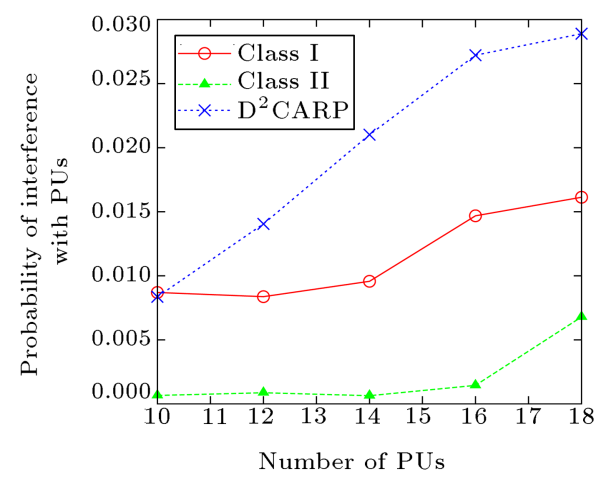

(a)

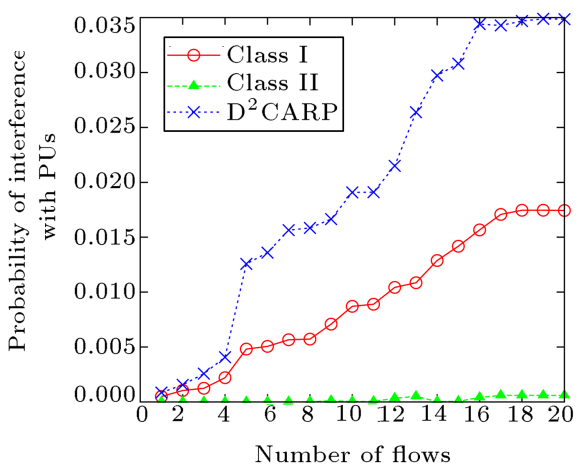

(b)

Figure 7. Probability of interference with PUs versus (a) the number of PUs, and (b) the number of flows. 


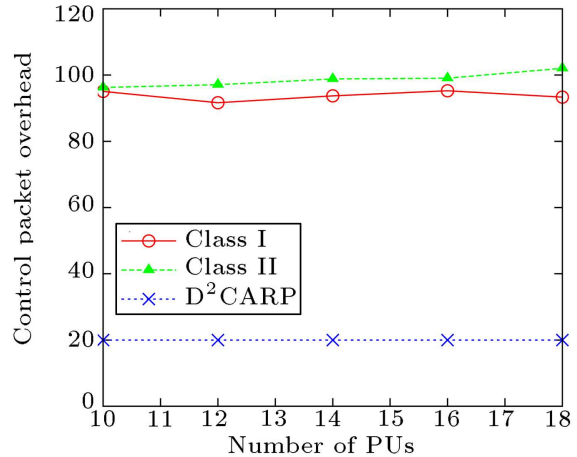

(a)

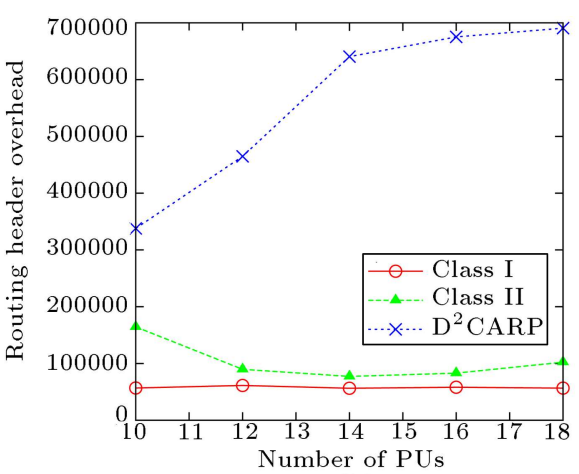

(b)

Figure 8. The number of PUs versus (a) the routing header overhead, and (b) the control packet overhead.

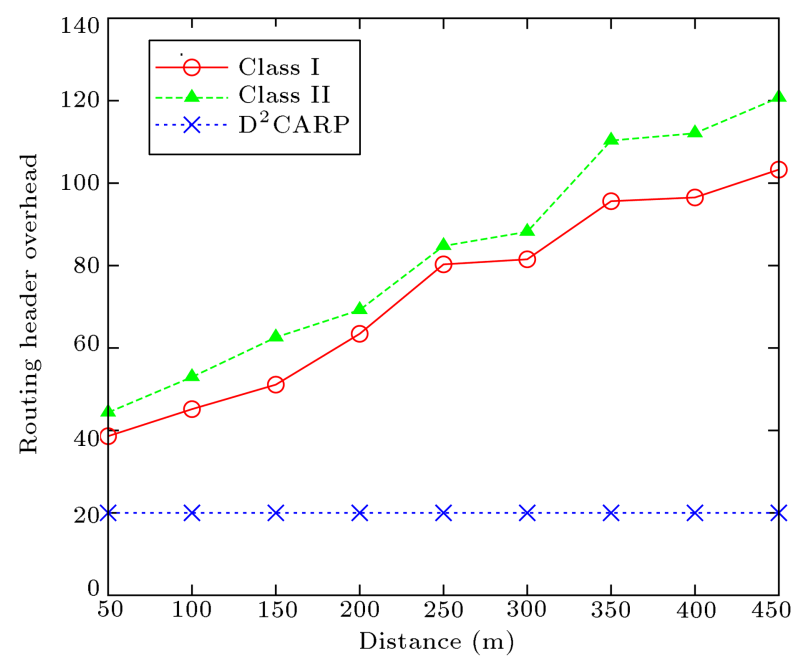

Figure 9. The source-destination distance versus routing header overhead.

header of packets in CMORC is bigger than that in $\mathrm{D}^{2} \mathrm{CARP}$. However, $\mathrm{D}^{2} \mathrm{CARP}$ sends RREQ packets over all available channels, while CMORC sends RREQ just over the CCC. Therefore, the number of control packets in $\mathrm{D}^{2} \mathrm{CARP}$ is more than CMORC.

Figure 8 clearly demonstrates the two overhead already explained. Routing header overhead of CMORC is almost 5 times bigger than that of $\mathrm{D}^{2} \mathrm{CARP}$, while the overhead of control packets in $\mathrm{D}^{2} \mathrm{CARP}$ is almost 5 to 6 times bigger than CMORC. Hence, there is a trade-off between these two overheads. As shown in Figure 9, the overhead of routing header in CMORC is proportional to the distance or path length. Hence, when the routes are long, the overhead of CMORC is more than $\mathrm{D}^{2} \mathrm{CARP}$. On the other hand, when the number of available channels increases, the overhead of control packet in $\mathrm{D}^{2} \mathrm{CARP}$ proportionally increases, while it is almost constant in CMORC because it sends control packets on all of these channels.

\subsection{Number of channels}

As the number of available channels increases, we usually expect more opportunity to exploit, and con- sequently, performance metrics often improve. Figure 10(a) shows the effect of the number of channels on end-to-end delay. As it is shown, class I routes always outperform the others. Interestingly, increasing the number of channels increases the end-to-end delay of class II routes. This happens because class II routes are optimized to reduce interference as far as possible and ignore other parameters completely. When the number of channels increases, there are paths that are more possible by different available channels and nodes. Class II routing strategy causes CMORC to find extremely long paths which almost avoid interference with PUs at the cost of more delay. Figure 10(b) illustrates how the number of channels influences PDR. As it is expected, increasing the number of channels increases PDR of all types of routes since it reduces interference and contention on all channels. As it is shown in Figure 11, probability of interference decreases as the number of channels increases. It is also intuitively obvious since the number of flows is constant. It should be noted that even class I route of CMORC, which is optimized to reduce delay, outperforms $\mathrm{D}^{2} \mathrm{CARP}$.

\subsection{Traffic distribution}

We have also investigated how traffic distribution can affect performance parameters. In our simulations, 30 different source nodes are sending packets to random destinations. Figure 12 shows how changing the percentage of class I packets can change PDR and end-to-end delay. The percentage shows how many flows are delay-intolerant (class I route) and how many are delay-tolerant (class II route). Hence, for routing protocols, such as $\mathrm{D}^{2} \mathrm{CARP}$, which do not consider these requirements, routing strategy for both flows would be similar. Class I packets in our routing protocol always face the least amount of end-to-end delay. When the number of class II flows is high, they encounter high end-to-end delay, since CMORC blindly chooses the routes that probably pass through the same regions with the least interference. Hence, the high contention in these regions causes delay. Since 


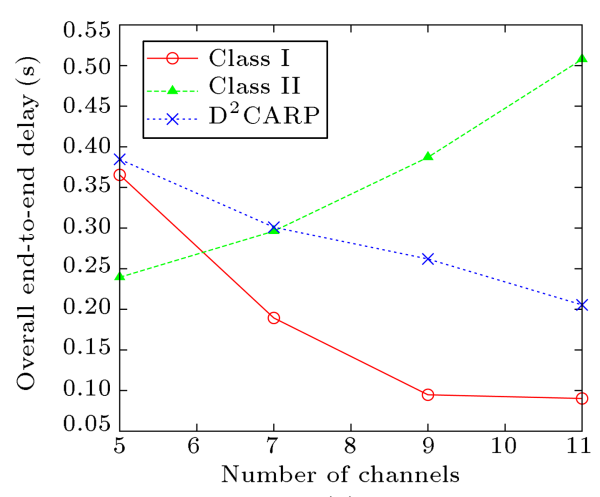

(a)

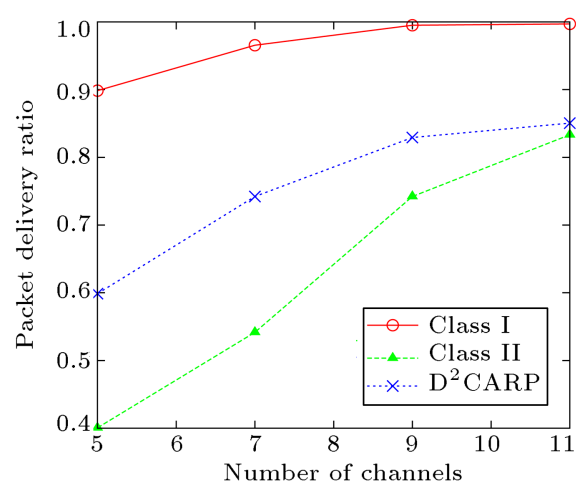

(b)

Figure 10. The number of channels versus (a) overall end-to-end delay, and (b) packet delivery ratio.

there is no different approach or mechanism available for these two types of traffic in $\mathrm{D}^{2} \mathrm{CARP}$, performance parameters should be almost the same, as shown in Figure 12.

As shown in Figure 12(b), the percentage of class I flows has a marginal effect on PDR. As it was investigated in Figure 6(b), PDR of class I routes in

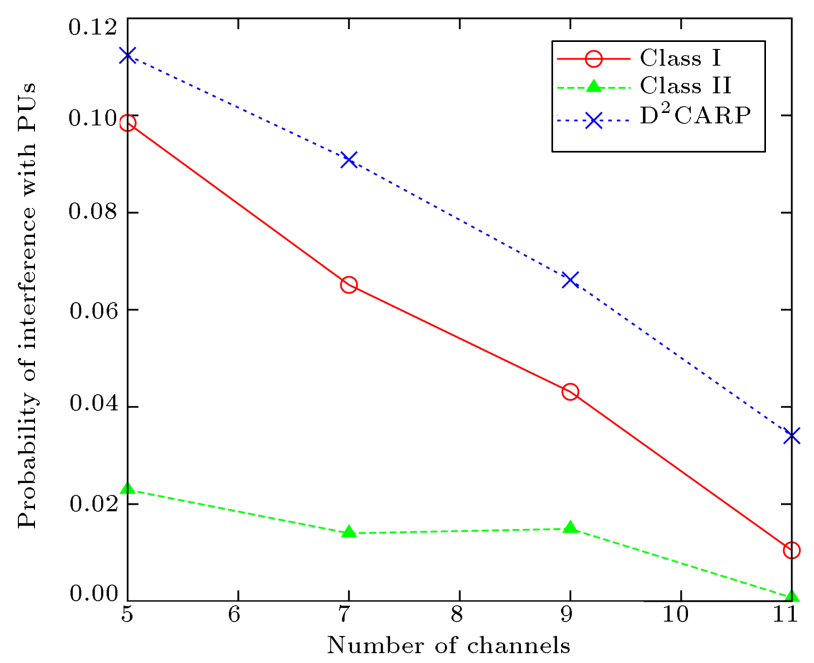

Figure 11. The source-destination distance versus probability of interference with PUs.

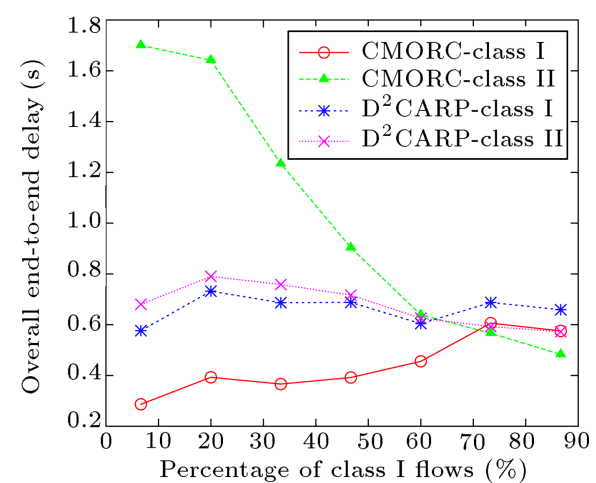

(a)
CMORC outperforms the others, and class II routes in CMORC have the worst PDR in most cases, except when there are only a few class II flows. Interestingly, no matter how many class II flows exist, class II flows in CMORC almost incur no interference in comparison with others, as depicted in Figure 13. However, the probability of interference of class II flows as well as

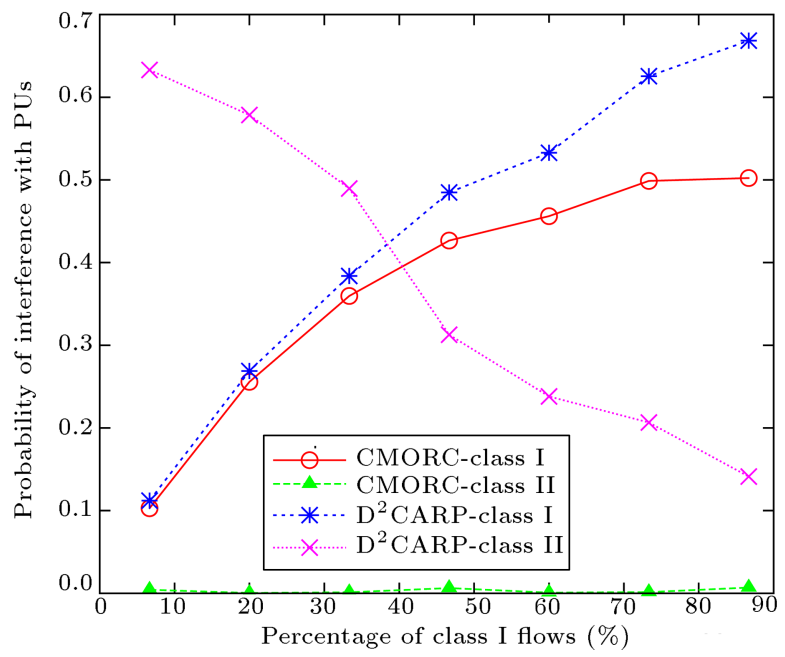

Figure 13. The percentage of class I flows versus probability of interference with PUs.

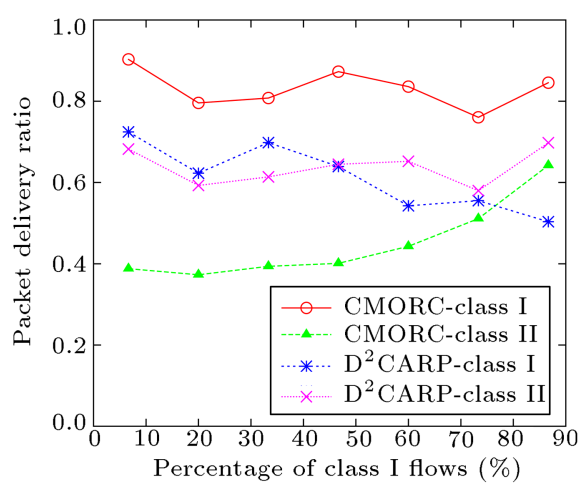

(b)

Figure 12. The percentage of class I flows versus (a) overall end-to-end delay, and (b) packet delivery ratio. 
$\mathrm{D}^{2} \mathrm{CARP}$ highly depends on the number of flows. As they increase, they incur more interference. However, class I flows in CMORC somehow avoid interference by indirectly using PUs behavior in their computation. That is why class I flows in CMORC often impose less interference than class I flows in $\mathrm{D}^{2} \mathrm{CARP}$.

\section{Conclusion and future research}

In this paper, we proposed a class-based multipath routing protocol, called CMORC, which is the first multipath routing protocol that takes into consideration both route discovery and rate allocation simultaneously. CMORC provides two classes of service to separately improve the end-to-end delay and probability of interference. Channel history in modeling the end-to-end delay for class I routes improves not only the end-to-end delay, but also the total interference. Class II routes also considerably reduce total interference, which is almost zero, while imposing higher delay. Moreover, the forwarding delay technique is used to let the route discovery stage find the better paths earlier. The efficiency of this technique is observed by simulation, and the technique is exploited in rate allocation stage to greatly reduce computational overhead. The performance of our proposed protocol is compared with that of $\mathrm{D}^{2} \mathrm{CARP}$ via simulations. Simulation results confirm the performance improvement of our protocol.

Although the end-to-end delay and interference with PUs are improved, delay estimation of CMORC might not always represent the real value of delay, since it is based on the assumption that arrival rate of intermediate nodes is independent of other nodes. Our future work will focus on modeling the end-to-end delay as a network of queue so that it estimates the real end-to-end value better.

\section{References}

1. Akyildiz, I.F., Lee, W.Y. and Chowdhury, K.R. "CRAHNs: Cognitive radio ad hoc networks", AD hoc Networks, 7 (5), pp. 810-836 (2009).

2. Chowdhury, K.R. and Akyildiz, I.F. "CRP: A routing protocol for cognitive radio ad hoc networks", IEEE Journal on Selected Areas in Communications, 29(4), pp. 794-804 (2011).

3. Trong, H.C., Lee, S. and Hong, C.S. "A routing protocol using a reliable and high-throughput path metric for multi-hop multi-rate ad hoc networks", Annals of Telecommunications-Annales des Tlcommunications, 67(5-6), pp. 269-284 (2012).

4. Gui, L., Zhong, X. and Zou, S. "Traffic assignment algorithm for multi-path routing in cognitive radio Ad Hoc networks", IEEE Wireless Communications and Networking Conference (WCNC), pp. 1168-1173 (2013).
5. Cesana, M., Cuomo, F. and Ekici, E. "Routing in cognitive radio networks: Challenges and solutions", Ad Hoc Networks, 9(3), pp. 228-248 (2011).

6. Tarique, M., Tepe, K.E., Adibi, S. and Erfani, S. "Survey of multipath routing protocols for mobile ad hoc networks", Journal of Network and Computer Applications, 32(6), pp. 1125-1143 (2009).

7. Cacciapuoti, A.S., Caleffi, M. and Paura, L. "Reactive routing for mobile cognitive radio ad hoc networks", Ad Hoc Networks, 10(5), pp. 803-815 (2012).

8. Rahman, M.A., Caleffi, M. and Paura, L. "Joint path and spectrum diversity in cognitive radio ad-hoc networks", EURASIP Journal on Wireless Communications and Networking, $2012(1)$, pp. 1-9 (2012).

9. Khanna, B. and Ramanathan, R. "November. Interference-aware multipath routing in a cognitive radio ad hoc network", IEEE Military Communications Conference (MILCOM), pp. 855-860 (2011).

10. Chen, P.Y., Cheng, S.M., Ao, W.C. and Chen, K.C. "Multi-path routing with end-to-end statistical QoS provisioning in underlay cognitive radio networks", IEEE Conference on Computer Communications Workshops (INFOCOM WKSHPS), pp. 7-12 (2011).

11. Lin, L., Wang, A.P., Zhou, X.W. and Miao, X.N. "Noncooperative differential game based efficiencyaware traffic assignment for multipath routing in CRAHN", Wireless Personal Communications, 62(2), pp. 443-454 (2012).

12. Lin, L. and Tian, J. "Stable-aware traffic assignment for multi-path routing in cognitive radio Ad Hoc networks", IEEE International Conference on CyberEnabled Distributed Computing and Knowledge Discovery (CyberC), pp. 511-514 (2011).

13. Yadav, R.N. and Misra, R. "Multipath routing protocols in cognitive radio networks", IEEE 2014 Annual India Conference (INDICON), pp. 1-6 (2014).

14. Bhattacharya, A., Ghosh, S.C. and Sinha, B.P. "Multipath routing in cognitive radio networks for multimedia communication using sample division multiplexing", IEEE Global Communications Conference (GLOBECOM), pp. 1097-1102 (2012).

15. Su, H. and Zhang, X. "Cross-layer based opportunistic MAC protocols for QoS provisionings over cognitive radio wireless networks", IEEE Journal on Selected Areas in Communications, 26(1), pp. 118-129 (2008).

16. Chowdhury, K.R. and Felice, M.D. "Search: A routing protocol for mobile cognitive radio ad-hoc networks", Computer Communications, 32(18), pp. 1983-1997 (2009).

17. Lee, S.J. and Gerla, M. "Split multipath routing with maximally disjoint paths in ad hoc networks", IEEE International Conference on Communications (ICC), pp. 3201-3205 (2001). 
18. Rezaei, S. and Hemmatyar, A.M.A. "Impact of jitter mechanisms on metric-based wireless routing protocols", IEEE International Conference on Wireless Communications, Signal Processing and Networking (WiSPNET), pp. 574-578 (2016).

19. Cassandras, C.G. and Lafortune, S., Introduction to Discrete Event Systems, Springer Science \& Business Media (2009).

20. Boyd, S. and Vandenberghe, L., Convex Optimization, Cambridge University Press (2004).

\section{Biographies}

Shahbaz Rezaei received his BSc degree in Computer Engineering from University of Science and Culture,
Tehran, Iran, in 2011, and MSc degree from the Sharif University of Technology, Tehran, Iran, in 2013. He is currently a PhD student at UC Davis. His research interests include mobile ad hoc networks, cognitive radio networks and performance modeling.

Ali Mohammad Afshin Hemmatyar received BSc, $\mathrm{MSc}$, and $\mathrm{PhD}$ degrees in Electrical Engineering from Sharif University of Technology, Tehran, Iran, in 1988, 1991 and 2007, respectively. Since 1991, he has joined Department of Computer Engineering at Sharif University of Technology, where he is currently an Assistant Professor. His research interests are Vehicular adhoc networks, wireless sensor networks, cognitive radio networks, and social networks. 